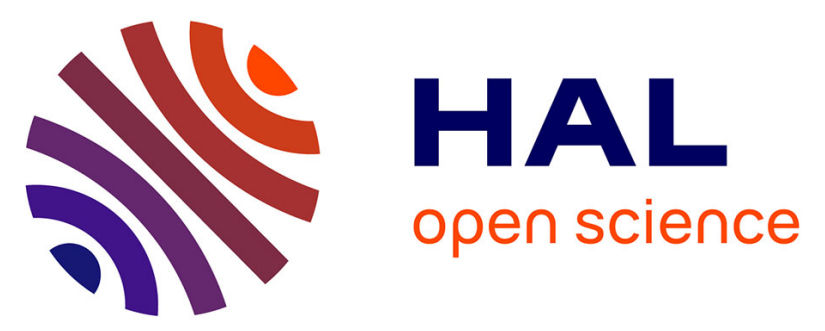

\title{
Early transmissible ampicillin resistance in zoonotic Salmonella enterica serotype Typhimurium in the late 1950s: a retrospective, whole-genome sequencing study Alicia Tran-Dien, Simon Le Hello, Christiane Bouchier, François-Xavier Weill
}

\section{- To cite this version:}

Alicia Tran-Dien, Simon Le Hello, Christiane Bouchier, François-Xavier Weill. Early transmissible ampicillin resistance in zoonotic Salmonella enterica serotype Typhimurium in the late 1950s: a retrospective, whole-genome sequencing study. The Lancet Infectious Diseases, 2018, 18 (2), pp.207-214. 10.1016/S1473-3099(17)30705-3 . pasteur-02019786

\section{HAL Id: pasteur-02019786}

https://hal-pasteur.archives-ouvertes.fr/pasteur-02019786

Submitted on 14 Feb 2019

HAL is a multi-disciplinary open access archive for the deposit and dissemination of scientific research documents, whether they are published or not. The documents may come from teaching and research institutions in France or abroad, or from public or private research centers.
L'archive ouverte pluridisciplinaire HAL, est destinée au dépôt et à la diffusion de documents scientifiques de niveau recherche, publiés ou non, émanant des établissements d'enseignement et de recherche français ou étrangers, des laboratoires publics ou privés.

\section{(c)(1)}

Distributed under a Creative Commons Attribution| 4.0 International License 
Early transmissible ampicillin resistance in zoonotic Salmonella enterica serotype Typhimurium in the late 1950s: a retrospective whole-genome sequencing study

Alicia Tran-Dien, M.Sc ${ }^{1}$, Simon Le Hello, Pharm.D ${ }^{1}$, Christiane Bouchier, Ph.D ${ }^{2}$, and François-Xavier Weill, M.D ${ }^{1 \ddagger}$

${ }^{1}$ Institut Pasteur, Unité des Bactéries Pathogènes Entériques, Centre National de Référence des Escherichia coli, Shigella et Salmonella, Paris 75015, France

${ }^{2}$ Institut Pasteur, Plate-forme Génomique (PF1), Paris, 75015, France

$\$$ Corresponding author

François-Xavier Weill: Centre National de Référence des Escherichia coli, Shigella et Salmonella, Unité des Bactéries Pathogènes Entériques, Institut Pasteur, 28 rue du docteur Roux, 75724 Paris cedex 15. Tel: +33-1 456883 45, Fax: +33-1 456888 37. E-mail: francois-xavier.weill@pasteur.fr

Running title: Early ampicillin resistance in Salmonella Typhimurium

Keywords: Salmonella Typhimurium, multidrug resistance, ampicillin, penicillins, betalactamase, antimicrobial resistance, emergence

Summary (254 words); main text (3780 words); four figures, two tables, one box (256 words), 30 references. Appendix with three pages. 


\section{SUMMARY}

\section{Background}

Ampicillin, the first semisynthetic penicillin active against Enterobacteriaceae, was released onto the market in 1961. The first outbreaks of disease caused by ampicillin-resistant Salmonella enterica serotype Typhimurium strains were identified in the United Kingdom in 1962 and 1964. This study was performed to date the emergence of this resistance in historical isolates of S. enterica serotype Typhimurium.

\section{Methods}

We studied a collection of 288 isolates collected between 1911 and 1969 from 31 countries on four continents. Antimicrobial drug susceptibility was tested for all isolates and wholegenome sequencing was carried out on a subset of 225 isolates. The mechanisms of resistance to ampicillin were identified.

\section{Findings}

Eleven isolates $(3.8 \%, 11 / 288)$ from three phylogenomic groups were resistant to ampicillin due to the acquisition of various beta-lactamase genes carried by different plasmids, including the virulence plasmid of $S$. enterica serotype Typhimurium. Three isolates producing TEM-1 were isolated in France $(n=1)$ and Tunisia $(n=2)$, in 1959 and 1960, respectively, before ampicillin went on sale. The vectors for ampicillin resistance were different from those reported in the strains responsible for the outbreaks in the United Kingdom in the 1960s.

\section{Interpretation}

The relationship between antibiotic use and the selection of resistance determinants is not as direct as often presumed. Our results suggest that the non-clinical use of narrow-spectrum 
penicillins (e.g., benzylpenicillin) may have favored the diffusion of bla $a_{\mathrm{TEM}-1}$ gene-carrying plasmids in the late 1950s in this prominent zoonotic bacterium.

\section{Funding}

Institut Pasteur, Santé publique France, the French government's Investissement d'Avenir programme, the Fondation Le Roch-Les Mousquetaires.

\section{Panel: Research in context}

\section{Evidence before this study}

We searched PubMed for articles published between January 1, 1900 and December 31, 1965, with the following search terms: "Salmonella and ampicillin and resistance", "Salmonella and penicillin and resistance", "Salmonella and penicillinase", and "Salmonella and betalactamase". We also searched for articles published between January 1, 1900 and August 31, 2017, with the following search terms "Salmonella and TEM", and "Salmonella and OXA". No language restrictions were used. We found no reports describing transmissible ampicillin resistance or beta-lactamase in Salmonella spp. isolates collected before 1962.

\section{Added value of this study}

We identified TEM-1 beta-lactamase-producing S. enterica serotype Typhimurium isolates, dating from 1959-1960, several years before the release of ampicillin onto the market. The genetic diversity of these ampicillin-resistant isolates, their resistance mechanisms and their geographic distribution suggest that ampicillin resistance had already diffused in this zoonotic pathogen at that time. We also found relative susceptibility of wild-type S. enterica serotype Typhimurium isolates to penicillin $\mathrm{G}(8 \mathrm{mg} / \mathrm{L})$, a narrow-spectrum penicillin widely used as a 
feed additive (subtherapeutic doses of antimicrobial agents over long periods of time) in livestock from the 1950s to the end of the 1960s.

\section{Implications of all the available evidence}

Our data shed light on the origin of resistance to a major antimicrobial agent in a prominent zoonotic bacterium. They show that environments in which penicillin $\mathrm{G}$ residues are abundant, such as farming environments in the late 1950s, can foster transmissible ampicillin resistance in S. enterica serotype Typhimurium. These findings highlight the need for a "onehealth" approach to tackling the issue of antibiotic resistance. 


\section{INTRODUCTION}

In May 2015, the 68th World Health Assembly endorsed a global action plan to tackle antimicrobial resistance, ${ }^{1}$ a global public health issue for the 21 th century. Eighty years ago, the discovery and commercialisation of "sulfa drugs" (sulfonamidochrysoidine and sulfanilamide) opened up a new era in the fight against bacterial infections, that of antibiotics. ${ }^{2}$ These synthetic bacteriostatic antibiotics were hailed as "magic bullets" or "wonder drugs", but were rapidly eclipsed by penicillin, a natural bactericidal substance from Penicillium rubrum that had been discovered earlier (1928) but did not go into mass production until World War 2 (WW2). ${ }^{2}$ In his princeps paper, Alexander Fleming noted that, unlike Gram-positive bacteria, the bacteria of the "coli-typhoid" group were not sensitive to penicillin. ${ }^{3}$ The first penicillin drug active against enterobacteria (broad-spectrum penicillin) was ampicillin, a semisynthetic derivative of 6-aminopenicillanic acid, ${ }^{4}$ commercialised by Beecham Research Laboratories under the name "Penbritin" in 1961. ${ }^{5}$ The first report describing beta-lactamases, encoded by "R factors" (plasmids), in Enterobacteriaceae strains with transmissible ampicillin-resistance, was published in $1965 .{ }^{6}$ In this report, two of the three Enterobacteriaceae strains studied were found to produce the TEM penicillinase. The $E$. coli isolate from Greece obtained in 1963 (the strain and its beta-lactamase were named TEM after the patient's name, Temoneria) ${ }^{7}$ and the S. enterica serotype Paratyphi B isolate obtained in the United Kingdom in 1963 were subsequently found to contain the bla $a_{\mathrm{TEM}-1 \mathrm{~A}}$ gene carried by a $\sim 40 \mathrm{~kb}$ IncX2 plasmid and a $100 \mathrm{~kb}$ IncF plasmid, respectively (Table 1). ${ }^{8,9}$ The third strain, a S. enterica serotype Typhimurium phage-type 1a (later reclassified as definitive phage-type [DT] 2), isolated in 1962 in Brighton, United Kingdom, produced a beta-lactamase with a substrate profile different from that of TEM (Table 1). This S. enterica serotype Typhimurium strain was isolated during the first reported outbreak caused by an 
ampicillin-resistant $S$. enterica serotype Typhimurium strain. It occurred in the second half of 1962, in the United Kingdom, at a time at which dozens of S. enterica serotype Typhimurium DT2 isolates resistant to ampicillin, streptomycin, sulfonamides and tetracyclines were found in humans and pigs. ${ }^{10}$ The plasmid of a representative strain, $\mathrm{R}_{1818}$, later renamed $\mathrm{R} 46,{ }^{9}$ was sequenced (GenBank accession no. AY046276) and found to be a $50 \mathrm{~kb}$ IncN plasmid carrying the $b l a_{\mathrm{OXA}-2}$ gene.

In 1963 and 1964, epidemic isolates of Salmonella enterica with transmissible ampicillinresistance were identified several times in Europe (Table 1). ${ }^{11-14}$ In particular, S. enterica serotype Typhimurium DT29 emerged following the adoption of intensive farming methods for the rearing of calves in the United Kingdom. ${ }^{12}$ In 1965, 1,297 and 576 isolates of this pathogen were obtained from cattle and humans, respectively. More than $96 \%(1,294 / 1,297$ and 555/576) of these isolates were multidrug-resistant. NTP1, the ampicillin resistance plasmid of representative strain $\mathrm{RT} 1,{ }^{13}$ was found to be a small $(8.3 \mathrm{~kb})$, multicopy, nonconjugative plasmid carrying the $b l a_{\mathrm{TEM}-1}$ gene. $^{14}$

This timeline suggests that the use of ampicillin may have been the primary cause of the selection of resistant isolates of this major foodborne pathogen.

With a view to improving our understanding of the emergence of resistance to ampicillin, one of the most widely used antibiotics to date, we studied a large collection of historical isolates of S. enterica serotype Typhimurium, a zoonotic agent that has remained one of the two predominant serotypes causing human disease in Europe since WW2. ${ }^{15,16}$ These 288 isolates were collected between 1911 and 1969, and 107 were obtained before ampicillin went on sale. 


\section{MATERIALS \& METHODS}

\section{Bacterial isolates}

The 288 S. enterica serotype Typhimurium isolates, analysed here were obtained from various sources (including humans, $n=149$; animals, food and feed, $n=113$ ) and geographic areas (including Europe, $n=161$; Africa, $n=90$; Asia, $n=19$; America, $n=15$ ), between 1911 and 1969 (table S1). They originated from the collections of the French National Reference Centre for E. coli, Shigella and Salmonella (FNRC-ESS) $(n=277)$, the Collection de l' Institut Pasteur (CIP) $(n=9)$, and the World Health Organization Collaborative Centre for Reference and Research on Salmonella $(n=2)$, Institut Pasteur, Paris, France. All isolates from the FNRC-ESS were maintained as stab cultures at room temperature. They have not been manipulated since being placed in storage (generally one to two weeks after their isolation from biological samples). All other isolates were stored as freeze-dried cultures.

\section{Antimicrobial drug susceptibility testing}

Antimicrobial drug susceptibility was determined by the disk diffusion method on MuellerHinton agar, with a panel of 32 antimicrobial drugs (Bio-Rad, Marnes-la-Coquette, France), as previously described. ${ }^{17}$ The minimal inhibitory concentrations (MICs) of ampicillin and penicillin $\mathrm{G}$ (also known as benzylpenicillin) of 67 isolates were determined with Etest strips (bioMerieux, Marcy L'Etoile, France) (table S1).

\section{Resistance transfer determination}

A resistance transfer experiment was carried out with the 11 ampicillin-resistant S. enterica serotype Typhimurium isolates on solid medium. E. coli $\mathrm{K}-12 \mathrm{~J} 5 \mathrm{Azi}^{\mathrm{R}}$ and S. enterica serotype Typhimurium STM 36-57 $\mathrm{Nal}^{\mathrm{R}}$, a nalidixic acid-resistant mutant generated in the 
laboratory from the wild-type isolate STM 36-57 (IncF-), were used as recipient strains for plasmid conjugative transfer screening, and for estimating transfer efficiency, respectively. Strains were mated on a filter placed on either Trypto-Casein-Soy (TCS) (when using $S$. enterica serotype Typhimurium STM 36-57 Nal ${ }^{\mathrm{R}}$ recipient strain) or Drigalski (when using $E$. coli $\mathrm{K}-12 \mathrm{~J} 5 \mathrm{Azi}^{\mathrm{R}}$ recipient strain) agar. Transconjugants were selected on the same type of agar medium as used for mating, but supplemented with either ampicillin $(75 \mathrm{mg} / \mathrm{L})$ or penicillin $\mathrm{G}(15 \mathrm{mg} / \mathrm{L})$ and nalidixic acid $(64 \mathrm{mg} / \mathrm{L})$ or sodium azide $(500 \mathrm{mg} / \mathrm{L})$. For isolates yielding no transconjugants on TCS agar, another mating experiment was performed on M9 minimal medium rather than TCS. ${ }^{18}$ Three transconjugants were selected at random for each experiment. Plasmid classification was based on PCR-based replicon typing. ${ }^{17}$ For matings between $S$. enterica serotype Typhimurium strains, CRISPOL typing (see below) was performed to distinguish between recipient and donor strains. Each conjugation experiment was carried out in triplicate. Conjugation frequencies were calculated by dividing the number of transconjugants (counted on agar medium containing nalidixic acid and ampicillin or penicillin $\mathrm{G}$ ) by the total number of recipient cells (counted on agar medium containing only nalidixic acid). The values shown are means from triplicate experiments.

\section{DNA extraction}

Total DNA was extracted with the Instagene kit (Biorad) or the Maxwell 16 cell DNA purification kit (Promega, Madison WI) for CRISPOL typing and whole-genome sequencing, respectively, in accordance with the manufacturer's recommendations.

\section{CRISPOL typing}


The CRISPOL (CRISpr POLymorphisms) high-throughput method for subtyping S. enterica serotype Typhimurium and its monophasic variant was carried out on the Luminex platform, as previously described. ${ }^{19}$

\section{Whole-genome sequencing}

Whole-genome sequencing was performed on a selection of 225 of the 288 S. enterica serotype Typhimurium isolates. The selection was designed to obtain wide coverage in terms of source, spatiotemporal and genetic diversity. Fifty-three $(84.1 \%, 53 / 63)$ isolates were not sequenced because they had a same CRISPOL type (CT) and were isolated in the same country within five years of those sequenced. The ten remaining non-sequenced isolates $(15.9 \%, 10 / 63)$ were isolated in the same country, within two years of those sequenced, but were mostly from unknown sources $(n=2)$ or sources already overrepresented (pets, rodents and birds, $n=6)$. The final selection consisted of 113/149 (75.8\%) isolates from humans; 93/113 (82.3\%) isolates from animals, food and feed; 132/161 (82.0\%) isolates from Europe; 58/90 (64.4\%) isolates from Africa; 19/19 (100\%) isolates from Asia; 13/15 (86.7\%) isolates from America; and 123/125 (98.4\%) of the CTs were represented (table S1). Whole-genome sequencing was carried out at the genomics platform of Institut Pasteur on an Illumina HiSeq 2500 platform generating 118 to 146 bp paired-end reads, yielding a mean of 233-fold coverage (minimum 41-fold, maximum 960-fold). Short-read sequence data were submitted to EnteroBase (http://enterobase.warwick.ac.uk/).

The oldest ampicillin-resistant S. enterica serotype Typhimurium isolate, ExPB 5-59 was also sequenced by GATC Biotech (Konstanz, Germany), on the PacBIO RS II platform (Pacific Biosciences, CA, USA). PacBio sequencing errors for homopolymers were corrected on the basis of Illumina sequences. The plasmid sequence was annotated with the RAST server 
(http://rast.nmpdr.org/), with manual inspection and correction. The sequence was deposited in GenBank under the accession code MF428416.

\section{Other studied genomes}

Two published S. enterica serotype Typhimurium genome sequences, those for DT104, ${ }^{20}$ a representative strain of the multidrug-resistant DT104 clone (GenBank accession no. NC_022569), and SO4698-09, ${ }^{21}$ a representative strain of the multidrug-resistant European monophasic clone (GenBank accession no. NZ_LN999997), were downloaded from GenBank, and 100 bp overlapping simulated reads were generated. Short-read sequences from the S. enterica serotype Weltevreden $840 \mathrm{~K}$ strain, used as the outgroup, were downloaded from EnteroBase.

\section{Read alignment and SNP detection}

Illumina-generated paired-end reads and simulated paired-end reads from publicly available assembled genomes were mapped onto the reference genome of $S$. enterica serotype Typhimurium strain LT2, including the chromosome (GenBank accession no. AE006468) and plasmid pSLT (GenBank accession no. AE006471), with Bowtie 2 version 2.1.0. Singlenucleotide polymorphisms (SNPs) were identified with SAMtools version 0.1.19, as previously described. ${ }^{22}$ SNPs present in insertion sequences identified by ISfinder (https://www-is.biotoul.fr/) or recombinogenic regions identified by Gubbins were removed, as previously described. ${ }^{22}$

\section{Phylogenetic analyses}

The maximum-likelihood (ML) phylogenetic tree was built from a 32,741-chromosomal SNP alignment of all S. enterica genomes. RAxML version 7.8.6 (GTRGAMMA substitution 
model) was used to construct the tree. We performed 100 bootstrap pseudoreplicate analyses to assess support for the ML phylogeny. The tree was rooted on $S$. enterica serotype Weltevreden $840 \mathrm{~K}$. The final tree was visualised with FigTree version 1.4.2.

\section{De novo assembly}

The reads for each strain were assembled de novo with SPAdes version 3.6.0. ${ }^{23}$

\section{Genetic analyses}

In silico MLST and serotyping were performed with the "Achtman 7 Gene" and "Serotype Prediction (SISTR)" tools of Enterobase, respectively. The presence and type of antimicrobial resistance genes (ARGs) or ARG-containing structures were determined with ResFinder version 2.1 (https://cge.cbs.dtu.dk/services/ResFinder/), PlasmidFinder $\quad$ version 1.3

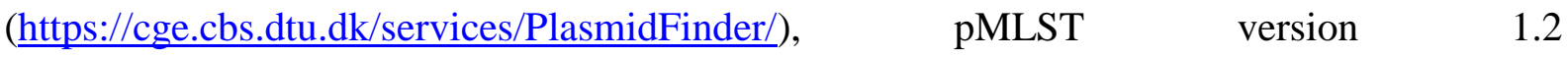
(https://cge.cbs.dtu.dk/services/pMLST/) on SPAdes assemblies. The CRISPOL Luminex data (presence or absence of 72 defined DNA sequences of 25-32 bp in length) were confirmed for each isolate, by searches for the 72 sequences within the whole-genome sequence (either from reads or the SPAdes assembly).

\section{ROLE OF THE FUNDING SOURCE}

The sponsor of the study had no role in study design, data collection, data analysis, data interpretation, or writing of the report. The corresponding author had full access to all the data in the study and had final responsibility for the decision to submit for publication.

\section{RESULTS}




\section{Antimicrobial drug susceptibility testing of historical isolates}

We carried out antimicrobial drug susceptibility testing on 288 historical S. enterica serotype Typhimurium isolates from various sources and geographic areas, between 1911 and 1969 (table S1). Most $(253 / 288,87.8 \%)$ of these isolates were susceptible to all the antibiotics tested (table S1). However, $11(3.8 \%, 11 / 288)$ were resistant to ampicillin, including $6(2.1 \%$, 6/288) also resistant to other antibiotics, such as sulfonamides, trimethoprim, tetracycline, and aminoglycosides (tables 2, S1, figure 1). These 11 isolates were collected from humans $(n=$ 10) in France $(n=8)$ and Tunisia $(n=3)$. Three $(2.8 \%, 3 / 107)$ of the isolates collected between 1911 and 1960 (i.e., before ampicillin went on sale) were resistant to ampicillin. These ampicillin-resistant isolates were collected from humans in France $(n=1)$ and Tunisia $(n=2)$, in 1959 and 1960, respectively.

\section{Population structure of the historical isolates}

The 225 historical S. enterica serotype Typhimurium isolates for which whole-genome sequences were obtained belonged to MLST eBurst groups 1 (98.7\%, 222/225), $138(0.4 \%$, $1 / 225)$ and $243(0.9 \%, 2 / 225)$. The most frequent sequence types (STs) were ST19 (89.3\%, 201/225), followed by ST313 and ST302 (1.3\%, 3/225 each). In total, 123 CRISPOL types were observed, with CT117 the most frequently represented $(4.9 \%, 11 / 225)$.

\section{Characterisation of the ampicillin-resistant isolates}

The 11 ampicillin-resistant S. enterica serotype Typhimurium isolates detected by the disk diffusion method and shown in Table 2 had penicillin MICs (for ampicillin or penicillin G) $\geq$ $256 \mathrm{mg} / \mathrm{L}$ (table S1, figure 2). The genomic analysis identified a beta-lactamase gene, either bla $a_{\mathrm{TEM}}(n=9)$ or bla $a_{\mathrm{OXA}}(n=2)$ in all these isolates. In a selection of $56 \mathrm{~S}$. enterica serotype Typhimurium isolates devoid of beta-lactamase genes and belonging to the different genomic 
clades of the collection (see below and Appendix, page 1), the MIC of ampicillin ranged from 0.06 to $8 \mathrm{mg} / \mathrm{L}$ (median $1 \mathrm{mg} / \mathrm{L}$ ), whereas the MIC of penicillin $\mathrm{G}$ ranged from 0.125 to 16 $\mathrm{mg} / \mathrm{L}$ (median $8 \mathrm{mg} / \mathrm{L}$ ) (table $\mathrm{S} 1$, figure 2).

The most frequent beta-lactamase gene, $b a_{\mathrm{TEM}-1 \mathrm{~B}}$, carried by transposon $\operatorname{Tn} 2,{ }^{26}$ was found in eight $(72.7 \%, 8 / 11)$ ampicillin-resistant $S$. enterica serotype Typhimurium isolates. The Tn2 transposon was located on plasmids from incompatibility groups $\operatorname{IncF}(n=5)$ and $\operatorname{IncX} 1(n=$ $3)$.

An analysis of $\operatorname{Tn} 2$ transposon junctions revealed that, in four isolates, $\operatorname{Tn} 2$ was located on pSLT, a $93 \mathrm{~kb}$ IncF plasmid known as the S. enterica serotype Typhimurium type strain LT2 virulence plasmid. ${ }^{18} \mathrm{Tn} 2$ was found integrated into three different sites of this pSLT (figure 3). In two ST313 isolates, STM 232-68 and STM 266-68, collected in France and Tunisia in 1968, the Tn2 integration site was identical to that of pSLT-BT, a multidrug resistance plasmid found in lineage II of the ST313 clone, a serotype Typhimurium population associated with invasive infections in Africa. ${ }^{25}$ Furthermore, as observed in pSLT-BT, a Tn21-like element was also found integrated into the Tn2 of the STM 232-68 and STM 26668 IncF plasmids. However, these two plasmids differed slightly from pSLT-BT in having a $6.8 \mathrm{~kb}$ IS 1 -mediated deletion (corresponding to region 41,666-48,543 of GenBank accession number FN432031) encompassing the integron (In2) region of the Tn21-like element. The IncF plasmid of the fifth isolate (STM 366-66) was derived from the NR1/R100 plasmid (lacking Tn21) (GenBank accession no. DQ364638). This isolate did not contain the pSLT virulence plasmid. 
The $45 \mathrm{~kb}$ Tn2-containing IncX1 plasmid pExPB5-59-1 was also sequenced with the PacBio platform. This plasmid was present in a clonal bacterial population (ST19-CT160) isolated in France over a 10-year period (1959 to 1969). The non-Tn2 backbone of plasmid pExPB5-591 resembled a $42.2 \mathrm{~kb}$ conjugative plasmid (pYU39_IncX; GenBank accession no. CP011431) found in an emerging invasive ST213 S. enterica serotype Typhimurium population from Mexico in the 2000s. In our study, five other S. enterica serotype Typhimurium isolates also carried an IncX1 plasmid but were devoid of antibiotic resistance genes (table S1). These plasmids had only $45-77 \%$ of their DNA sequences (98-99\% nucleotide identity) in common with pExPB5-59-1. Interestingly, the oldest of these isolates was collected during a foodborne outbreak of disease in Newcastle, United Kingdom in 1911.

The three $S$. enterica serotype Typhimurium isolates collected in France and Tunisia before the release of ampicillin contained the Tn2 transposon, in the $\operatorname{IncX} 1(n=1)$ and pSLT-derived $\operatorname{IncF}(n=2)$ plasmids. Tn2 was found inserted at two different sites within IncF.

\section{Transfer of ampicillin resistance}

The 11 ampicillin-resistant $S$. enterica serotype Typhimurium isolates were crossed with $S$. enterica serotype Typhimurium STM 36-57 $\mathrm{Nal}^{\mathrm{R}}$ recipient strains in the presence of ampicillin $(75 \mathrm{mg} / \mathrm{L})$, to determine the transferability of the different bla gene-carrying plasmids. The nine isolates containing a bla $a_{\mathrm{TEM}}$ gene-carrying plasmid were found to transfer ampicillin resistance by conjugation. Transfer frequencies ranged from $2.4 \times 10^{-4}$ (IncI1 plasmid from isolate STM 127-67) to $1.6 \times 10^{-8}$ (pSLT-derived IncF plasmid from isolate STM 266-68) transconjugants/recipient (Appendix, page 2). The use of M9 minimal medium was required to obtain transconjugants for STM 110-60. The two isolates containing a bla $a_{\text {OXA }}$ 
gene (STM 84-67 and STM 345-66) did not transfer their ampicillin resistance by conjugation.

The four Tn2-carrying plasmids tested (the IncX1 plasmid from isolate ExPB 5-59, pSLTderived IncF plasmids from isolates STM 65-60 and STM 110-60, and NR1/R100-derived IncF plasmids from isolate STM 366-66) were transferred between the two strains of $S$. enterica serotype Typhimurium in the presence of penicillin $G(15 \mathrm{mg} / \mathrm{L})$. Transfer frequencies ranged from $1.2 \times 10^{-3}$ (IncX1 plasmid from isolate ExPB 5-59) to $3.0 \times 10^{-6}$ (pSLT-derived IncF plasmid from isolate STM 110-60) transconjugants/recipient.

\section{Genetic relationships between the ampicillin-resistant isolates}

A whole-genome phylogeny based on 32,741 SNPs evenly distributed over the non-repetitive non-recombinant core genome showed the clustering of the 11 ampicillin-resistant S. enterica serotype Typhimurium isolates into three groups (figure 4, Appendix, page 3).

The main group comprised seven ampicillin-resistant isolates collected in France between 1959 and 1969, with four types of beta-lactamase genes (bla $a_{\mathrm{TEM}-1 \mathrm{~A}}, b l a_{\mathrm{TEM}-1 \mathrm{~B}}, b l a_{\mathrm{OXA}-1}$, and $b\left(a_{\mathrm{OXA}-2}\right)$. These seven ampicillin-resistant isolates belonged to a clade consisting of 13 West and South European isolates (plus one North African isolate) collected from humans, food and animals (chicks) and belonging to ST19 and CT46 or variants of CT46 (table S1).

The second group of ampicillin-resistant isolates comprised two human ST19-CT896 isolates collected in Tunisia in 1960. They belonged to a clade consisting of 14 ST19-CT117 (or CT117-derived) isolates mostly obtained from humans (with the exception of one isolate from a sausage) in France, Belgium and Tunisia between 1953 and 1963. 
The third group comprised the two ampicillin-resistant ST313-CT810 isolates collected in France and Tunisia in 1968. They belonged to a clade consisting of six isolates belonging to either ST302 $(n=3)$ or ST313 $(n=3)$. The four non ampicillin-resistant isolates originated from Turkey (two ST302 and one ST313) and Tunisia (one ST302) and were collected between 1956 and 1965.

\section{DISCUSSION}

We show here that transmissible ampicillin resistance actually emerged several years earlier than previously thought in $S$. enterica serotype Typhimurium, as the beta-lactamase bla $_{\mathrm{TEM}-1 \mathrm{~B}}$ gene was found on two different plasmids (IncX1 and IncF) in three isolates from Europe (France) and Africa (Tunisia) collected in 1959-1960. However, we did not aim here to determine the true extent of the global distribution of ampicillin resistance in S. enterica serotype Typhimurium, due to an obvious sampling bias (i.e., the available historical isolates studied here were predominantly from France and former French colonies in Africa and Asia). Another limitation of our study was the mode of storage of the collection strains. Almost all the isolates were stored as stab cultures and the absence of antibiotic selection pressure may not have favored the maintenance of acquired plasmids in the long term in these strains, as

previously reported for Bacillus anthracis. ${ }^{26}$ The proportion of ampicillin-resistant isolates in our selection of Salmonella isolates may therefore be underestimated.

Nevertheless, the use of ampicillin, the first broad-spectrum penicillin indicated for the treatment of infections due to Enterobacteriaceae, which began in $1961,{ }^{5}$ is unlikely to have led to the selection of these early ampicillin-resistant isolates. Following the emergence of 
multidrug resistance in zoonotic S. enterica serotype Typhimurium DT29, E.S. Anderson from the Central Public Health Laboratory in the United Kingdom warned that the use of penicillin $\mathrm{G}$ and tetracyclines as feed additives for livestock (in poultry and pigs) and as prophylactics in cattle was responsible for the emergence of these multidrug-resistant $S$. enterica serotype Typhimurium isolates. ${ }^{13}$ Long-term exposure to subtherapeutic levels of antibiotics in livestock may have been critical for the selection of ampicillin resistance in a prevalent zoonotic agent found in a wide range of food-producing animals. We found that the median MIC of penicillin G in wild-type S. enterica serotype Typhimurium isolates was not particularly high, at $8 \mathrm{mg} / \mathrm{L}$, whereas Fleming, in his rudimentary assays based on Penicillium broth filtrates, reported that members of the "coli-typhoid group" were unaffected by the inhibitory power of penicillin. ${ }^{3}$ The relatively low MIC values obtained for penicillin G against $S$. enterica serotype Typhimurium may account for the acquisition of ampicillin resistance in an environment in which penicillin $\mathrm{G}$ residues reached concentrations just above the MICs of the bacteria. For example, in France during the 1960s, penicillin G was permitted as a food additive, and was used at concentrations of 5-200 $\mathrm{mg} / \mathrm{kg}$ of feed, in all animals for human consumption. ${ }^{27}$ Penicillin $\mathrm{G}$ is sensitive to gastric acid, but its poor absorption by the digestive system can result in non-negligible concentrations in the digestive tract and excretion onto the litter. A study in the US in 1975 found that the litter of broiler chickens fed with antibiotics contained 0 to $25 \mathrm{mg} / \mathrm{kg}$ (mean $12.5 \mathrm{mg} / \mathrm{kg}$ ) penicillin. ${ }^{28}$ Using similar concentrations (15 mg/L of penicillin G), we showed that a bla gene carried by IncX1 or IncF plasmids could be successfully transferred between $S$. enterica serotype Typhimurium strains. Our results provide no proof of a causal link between the use of penicillin $G$ and the emergence of transmissible ampicillin-resistance in livestock, but they suggest that an environment in which penicillin $\mathrm{G}$ residues were abundant, such as the farming environment described by Anderson ${ }^{22}$, may have favored the diffusion of bla gene-carrying plasmids in the 
most prevalent Salmonella serotype at the time. We identified no ampicillin-resistant isolates originating from livestock. However, this may reflect the fact that less than 50\% (39/96) of the animal isolates tested were from livestock, with $\sim 40 \%(16 / 39)$ of the livestock isolates originating from Africa and South America. After 1969, in the United Kingdom, in accordance with the recommendations of the joint committee on the use of antibiotics in animal husbandry and veterinary medicine (known as the "Swann" Committee in honor of its chairman), the use of growth promoters in livestock was eventually restricted to drugs with little or no application as therapeutic agents in humans or animals, and penicillins, in particular, were identified as unsuitable for use as growth promoters.

Interestingly, the vectors of ampicillin resistance in our S. enterica serotype Typhimurium isolates, mostly from France, differed from those implicated in the first outbreaks in the United Kingdom, despite the geographic proximity of France and the United Kingdom. Thus, the early emergence of ampicillin resistance in S. enterica serotype Typhimurium was not due to a single expansion of a clonal population that had acquired a particular plasmid encoding a beta-lactamase, but to multiple independent acquisitions of bla $a_{\mathrm{TEM}}$ gene-carrying plasmids by different bacterial populations followed by diffusion of various extents within individual countries, from the very rare isolations of CT160 over a 10-year period in France to the hundreds of isolations of DT29 per year in the United Kingdom. This situation contrasts with the rapid global dissemination subsequently observed for two multidrug-resistant S. enterica serotype Typhimurium clones: DT104 in the 1980s and the monophasic 1,4,[5],12:i:- in the 2000 s. $^{29,30}$ This difference may be due to changes in the food trade, which was much less globalised in the late 1950s-early 1960s than it has been since in the 1980s. 
In conclusion, our study sheds light on the early steps in the emergence of resistance to ampicillin, the first penicillin active against Enterobacteriaceae, still in widespread use. We found that the relationship between antibiotic use and the selection of resistance determinants was not as direct as often presumed. Since the early days of antibiotic use, agriculture has played a role in the selection of resistant pathogens alongside medicine, particularly during periods of intensification. These findings highlight the need for a "one-health" approach to tackling the issue of antibiotic resistance. 
Table 1: Characteristics of the first S. enterica (and E. coli TEM) isolates with plasmidmediated ampicillin resistance to be reported.

\begin{tabular}{|c|c|c|c|c|c|c|c|}
\hline Bacterial host & Origin & AST & Plasmid & Type & bla gene & $\begin{array}{c}\text { Other resistance } \\
\text { genes }\end{array}$ & Reference \\
\hline $\begin{array}{c}S . \text { Typhimurium } \\
\text { DT2 }\end{array}$ & $\begin{array}{c}\text { 1962, UK, } \\
\text { Humans and } \\
\text { pigs }\end{array}$ & ApStrSulTe & $\mathrm{R}_{1818}=\mathrm{R} 46^{*}$ & $\begin{array}{l}51 \mathrm{~kb} \\
\text { IncN }\end{array}$ & OXA- $2^{\Omega}$ & $\operatorname{aadA1}, \operatorname{sull}, \operatorname{tet}(\mathrm{C})$ & 6,10 \\
\hline S. Paratyphi B & $\begin{array}{l}\text { 1963, UK, } \\
\text { Human }\end{array}$ & ApKCSul & $\mathrm{R}_{7268}=\mathrm{R} 1^{\dagger}$ & $\begin{array}{l}97.5 \mathrm{~kb}, \\
\text { IncF }\end{array}$ & TEM-1A & $\begin{array}{l}\operatorname{aadAl}, \operatorname{aph}\left(3^{\prime}\right)-I c, \\
\text { catAl,sul1 }\end{array}$ & 6,8 \\
\hline E. coli & $\begin{array}{c}\text { 1963, Greece, } \\
\text { Human }\end{array}$ & ApStr & $\mathrm{R}_{\mathrm{TEM}}=\mathrm{R} 6 \mathrm{~K}^{\#}$ & $\begin{array}{l}39 \mathrm{~kb}, \\
\text { IncX2 }\end{array}$ & TEM-1A & $\operatorname{str} A / B$ & 6,9 \\
\hline$S$. Derby & $\begin{array}{l}\text { 1963, } \\
\text { France, } \\
\text { Humans }\end{array}$ & ApStrKCSulTe & $\mathrm{R}_{4-5}$ & 10 & - & - & 11 \\
\hline S. Oranienburg & $\begin{array}{c}\text { 1964, } \\
\text { France, } \\
\text { Humans }\end{array}$ & ApStrKCSulTe & $\mathrm{R}_{7}$ & - & - & - & 11 \\
\hline $\begin{array}{l}S . \text { Typhimurium } \\
\text { DT29 }\end{array}$ & $\begin{array}{l}\text { 1964, UK, } \\
\text { Humans and } \\
\text { cattle }\end{array}$ & ApStrSulTe & $\mathrm{NTP} 1^{\mathrm{NC}}$ & $8.3 \mathrm{~kb}$ & TEM-1 & None & $12-14$ \\
\hline
\end{tabular}

AST, antimicrobial drug susceptibility type; Ap, ampicillin; Str, streptomycin; K, kanamycin; C,

chloramphenicol; Sul, sulfonamides; Tmp, trimethoprim; Te, tetracycline; -, unknown; DT, definitive phagetype; available from GenBank under accession nos. AY046276* or KY749247 $7^{\dagger}$ or from the Wellcome Trust Sanger Institute ${ }^{\#},{ }^{N C}$ non-conjugative; ${ }^{\Omega}$ duplicated gene.

Table 2: Characteristics of the 11 ampicillin-resistant S. enterica serotype Typhimurium isolates identified in this study.

\begin{tabular}{|c|c|c|c|c|c|c|c|c|c|}
\hline Isolate & Year & Country & Host & AST & bla gene & $\begin{array}{l}\text { Other resistance } \\
\text { genes }\end{array}$ & ST & CT & Plasmid \\
\hline ExPB 5-59 & 1959 & France & Human & Ap & TEM-1B & - & 19 & 160 & $\operatorname{IncX} 1^{\dagger \oslash}$ \\
\hline STM $110-60^{*}$ & 1960 & Tunisia & Human & ApStrSpSul & TEM-1B & $\operatorname{aad} A 1$ & 19 & 896 & $\operatorname{IncF} F^{\dagger}$ \\
\hline STM 65-60* & 1960 & Tunisia & Human & Ap & TEM-1B & - & 19 & 896 & $\operatorname{IncF}^{\dagger}$ \\
\hline STM 345-66 & 1966 & France & Human & ApStrSpSulTe & OXA-2 & 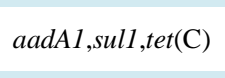 & 19 & 46 & $\begin{array}{l}\text { IncI1, } \\
\text { IncN }^{\Omega}\end{array}$ \\
\hline STM 366-66 & 1966 & France & Human & ApSul & TEM-1B & sull & 19 & 46 & 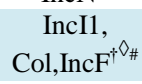 \\
\hline ExPB 63-67 & 1967 & France & Human & Ap & TEM-1B & - & 19 & 160 & $\operatorname{IncX} 1^{\dagger}$ \\
\hline STM 84-67 & 1967 & France & Human & ApSpKCSulTe & OXA-1 & $\begin{array}{c}a p h\left(3^{\prime}\right)-I a, \\
\text { aadAl,catA1,sul1, } \\
\text { tet(B) }\end{array}$ & 19 & 46 & $\mathrm{Col}, \mathrm{IncF}$ \\
\hline STM 127-67 & 1967 & France & Human & ApK & TEM-1A & $a p h\left(3^{\prime}\right)-I a$ & 19 & 46 & $\operatorname{IncI} 1^{\dagger}$ \\
\hline STM 232-68 & 1968 & France & Human & ApStrCSulTmp & TEM-1B & $\begin{array}{c}\text { strA/B,catAl,sul2, } \\
\text { dfrAl }\end{array}$ & 313 & 810 & $\operatorname{IncF}^{\dagger}$ \\
\hline TM 266-68 & 1968 & Tunisia & $?$ & ApStrCSulTmp & TEM-1B & $\begin{array}{c}\text { strA/B,catAl,sul2 } \\
\text { dfrAl }\end{array}$ & 313 & 810 & $\operatorname{Inc} F^{\dagger}$ \\
\hline ExPB 37-69 & 1969 & France & Human & Ap & TEM-1B & - & 19 & 160 & $\operatorname{IncX} 1^{\dagger}$ \\
\hline
\end{tabular}

AST, antimicrobial drug susceptibility type; Ap, ampicillin; Str, streptomycin; Sp, spectinomycin; K, kanamycin; C, chloramphenicol; Sul, sulfonamides; Tmp, trimethoprim; Te, tetracycline; ?, unknown; *, both isolates were received, without associated epidemiological information, from the Pasteur Institute of Tunis in August (STM 65-60) and October (STM 110-60) 1960, together with other isolates of Salmonella spp., Shigella spp. and enteropathogenic E. coli; plasmid location of the beta-lactamase gene demonstrated by conjugation ${ }^{\dagger}$ and/or genomic analysis ${ }^{\diamond}{ }^{\Omega}$ incomplete IncN plasmid ( $8.8 \mathrm{~kb}$ fragment similar to R46, comprising the region from $\operatorname{ardA}$ to repA); ${ }^{\text {IncF }}$ plasmid derived from NR1/R100 lacking Tn21, the other IncF plasmids are derived from virulence plasmid pSLT. 


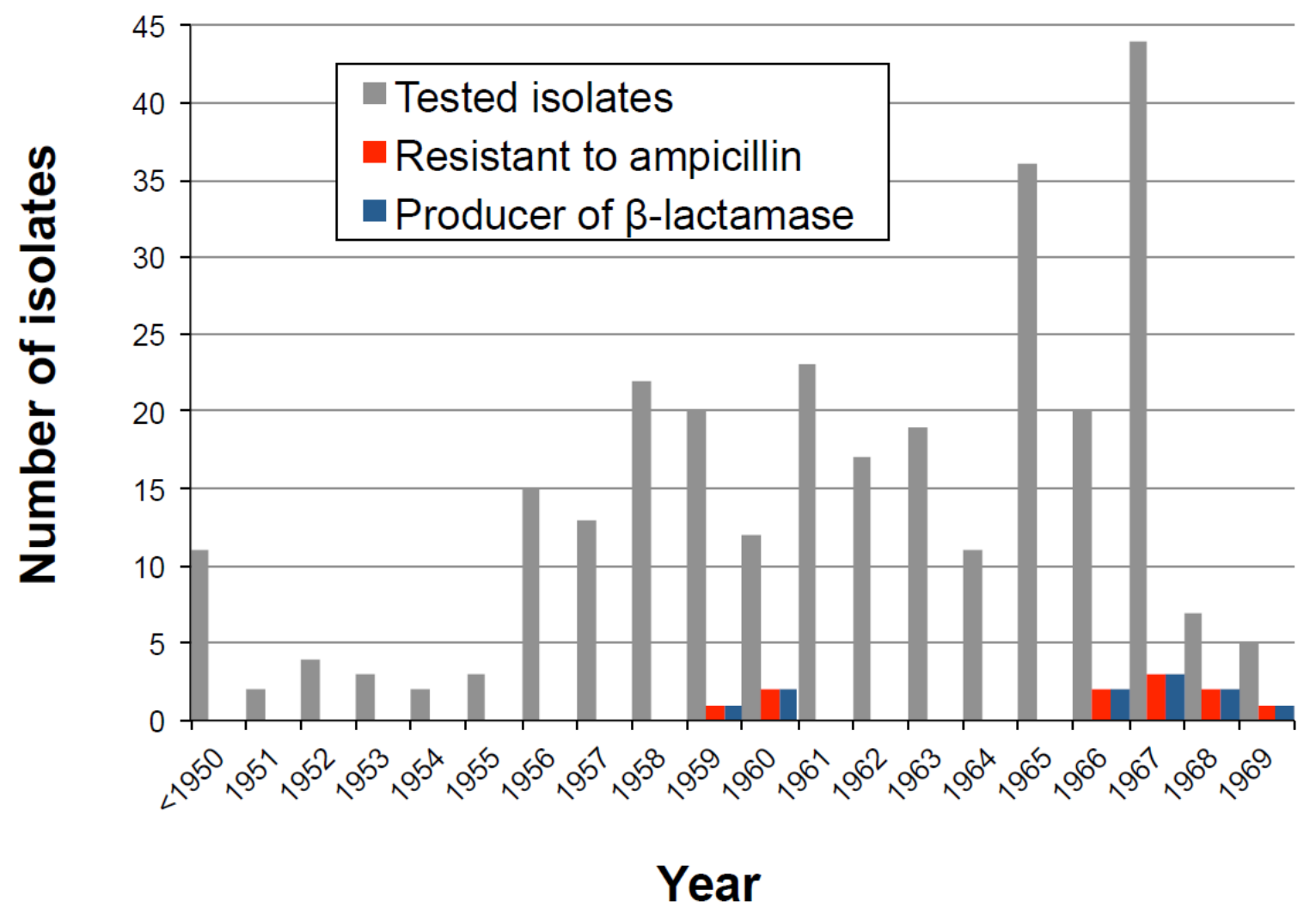

Figure 1. Number of ampicillin-resistant and beta-lactamase-producing $S$. enterica serotype Typhimurium isolates, by year of isolation.

The number of $S$. enterica serotype Typhimurium isolates studied is shown in gray, that of isolates resistant to ampicillin is shown in red, and that of isolates producing a beta-lactamase is shown in blue. 


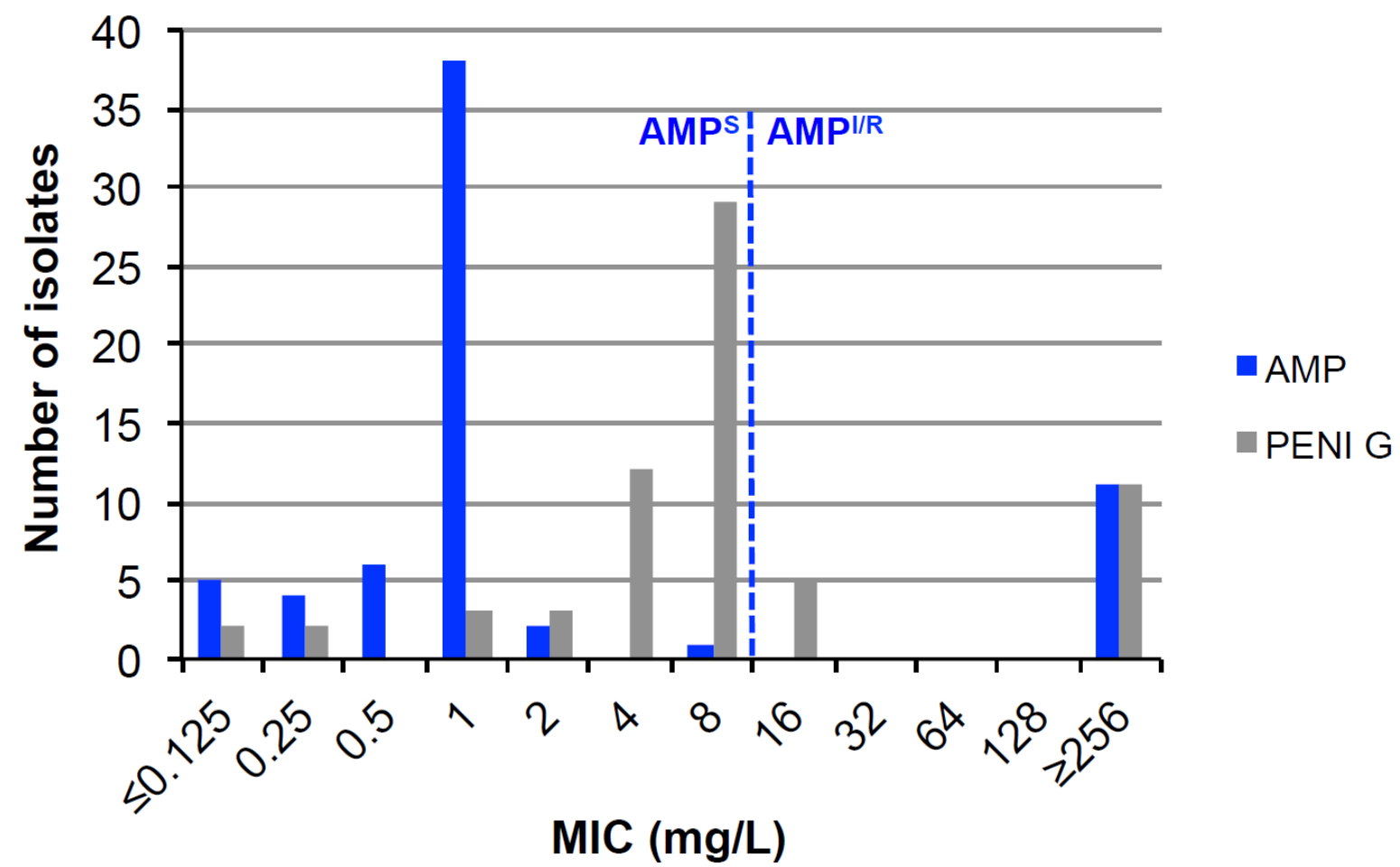

Figure 2: The distribution of ampicillin and penicillin G MICs in a selection of $67 S$. enterica serotype Typhimurium isolates.

The clinical breakpoint distinguishing ampicillin susceptibility from ampicillin nonsusceptibility according to EUCAST ( $\mathrm{S} \leq 8 \mathrm{mg} / \mathrm{L} ; \mathrm{R}>8 \mathrm{mg} / \mathrm{L})$ and CLSI $(\mathrm{S} \leq 8 \mathrm{mg} / \mathrm{L} ; \mathrm{I}=16$ $\mathrm{mg} / \mathrm{L} ; \mathrm{R} \geq 32 \mathrm{mg} / \mathrm{L}$ ) is shown by a dashed blue bar. 


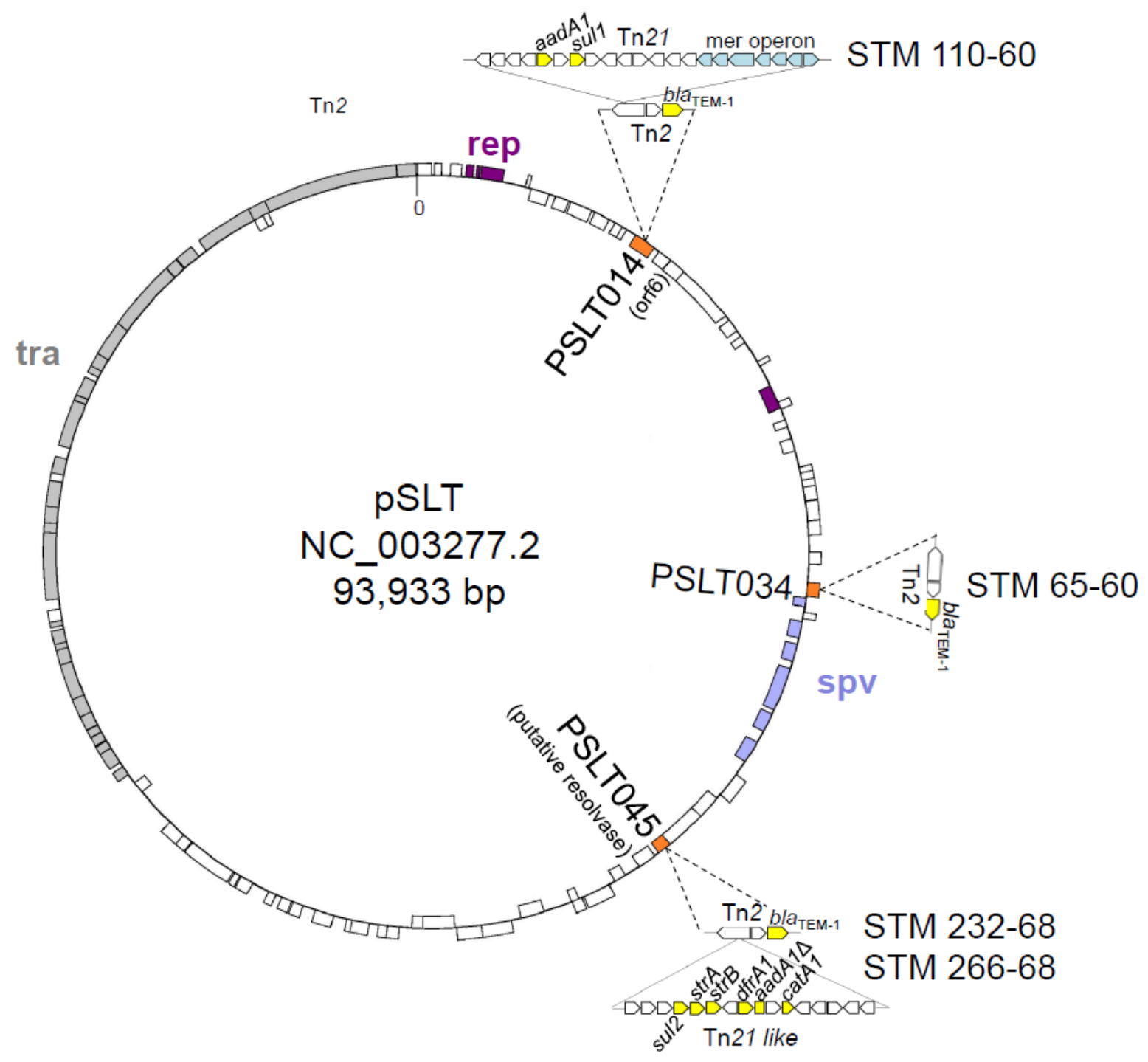

Figure 3: Integration sites of the $\mathrm{Tn} 2$ transposon within the IncF virulence plasmid pSLT in five ampicillin-resistant $S$. enterica serotype Typhimurium isolates.

The genes into which Tn2 is integrated are boxed in red and their names and function (in parentheses), according to GenBank accession no. NC_003277.2, are shown. The antibiotic resistance genes are boxed in yellow. The plasmid genes involved in transfer (tra), replication (rep) and virulence (spv) are shown in gray, purple and light blue, respectively. 


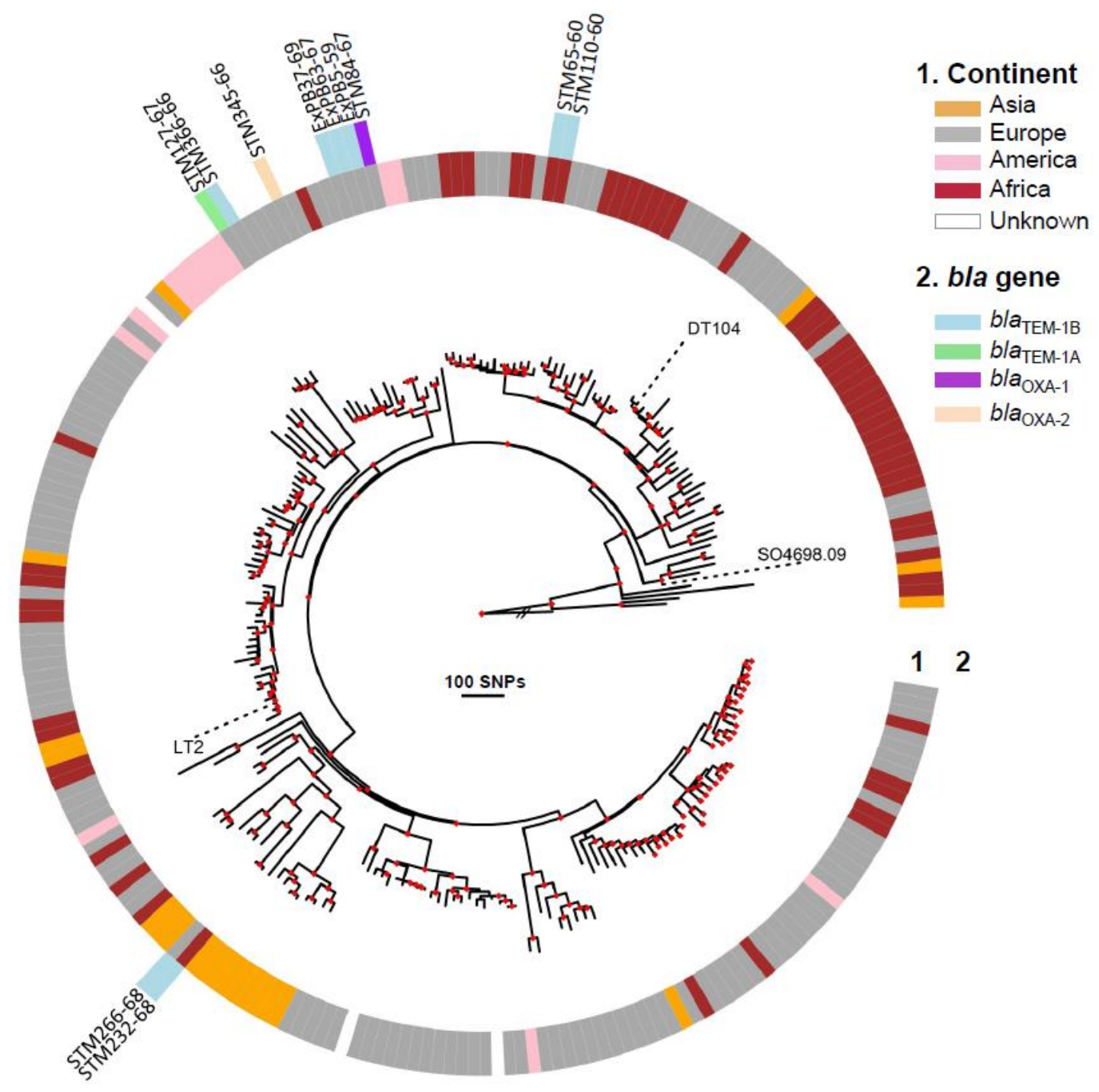

Figure 4: Maximum-likelihood phylogeny of the $S$. enterica serotype Typhimurium isolates.

The geographic origin (continent) of the isolates is shown in ring 1. The beta-lactamase genes of the 11 ampicillin-resistant isolates are indicated in ring 2 . The beta-lactamase genes of comparison genomes DT104 $\left(\right.$ bla $\left._{\mathrm{PSE}-1}=b l a_{\mathrm{CARB}-2}\right)$ and monophasic $\left(b l a_{\mathrm{TEM} 1-\mathrm{B}}\right)$ are not shown. The names of the 11 ampicillin-resistant isolates are indicated above ring 2. The LT2, DT104 
and monophasic (SO4698-09) comparison genomes are indicated by black dashed lines. The nodes supported by bootstrap values $\geq 0.9$ are indicated by red diamonds. The $S$. enterica serotype Weltevreden $840 \mathrm{~K}$ strain was used as the outgroup, and its branch length has been shortened by a factor of 100 , as indicated by the double slash (//), to improve visualisation. 


\section{AUTHOR CONTRIBUTIONS}

Conceived and designed the experiments: FXW. Performed the experiments: ATD.

Contributed reagents/materials/analysis tools: SLH, CB. Analysed the data: ATD and FXW.

Wrote the paper: ATD and FXW. Reviewed, critiqued and offered comments on the text:

SLH, CB.

\section{ACKNOWLEDGMENTS}

We thank G. Costilhes, L. Ma, and A. Rivasseau for expert technical assistance. We thank all corresponding laboratories of the French National Reference Centre for E. coli, Shigella, and Salmonella.

The French National Reference Centre for E. coli, Shigella, and Salmonella is funded by the Institut Pasteur and Santé Publique France. A. Tran-Dien was supported by a grant from the Fondation Le Roch-Les Mousquetaires. The "Unité des Bactéries Pathogènes Entériques" belongs to the "Integrative Biology of Emerging Infectious Diseases" Laboratory of Excellence funded by the French Government "Investissement d'Avenir" programme (grant no. ANR-10-LABX-62-IBEID). The Genomics Platform is member of the "France Génomique" consortium (ANR10-INBS-09-08).

\section{CONFLICTS OF INTEREST}

None of the authors has any competing interests or conflicts of interest to declare. 


\section{REFERENCES}

1. WHO. Global action plan on antimicrobial resistance. Geneva: World Health Organization, 2015. Available from:

http://www.wpro.who.int/entity/drug_resistance/resources/global_action_plan_eng.pdf (accessed June 26th 2017).

2. Bud R. Penicillin: Triumph and Tragedy. Oxford University Press, Oxford 2007.

3. Fleming A. On the antibacterial action of cultures of a Penicillium, with special reference to their use in the isolation of B. influenza. Br J Exp Pathol 1929; 10: 226236.

4. Batchelor FR, Doyle FP, Nayler JH, Rolinson GN. Synthesis of penicillin: 6aminopenicillanic acid in penicillin fermentations. Nature 1959; 183:257-8.

5. Knox R. A survey of new penicillins. Nature 1961; 192:492-6.

6. Datta N, Kontomichalou P. Penicillinase synthesis controlled by infectious R factors in Enterobacteriaceae. Nature 1965; 208:239-41.

7. Medeiros AA. Beta-lactamases. Br Med Bull 1984; 40:18-27.

8. Hedges RW, Datta N, Kontomichalou P, Smith JT. Molecular specificities of R factordetermined beta-lactamases: correlation with plasmid compatibility. J Bacteriol 1974; 117:56-62.

9. Cox KEL, Schildbach JF. Sequence of the R1 plasmid and comparison to F and R100. Plasmid 2017; 91:53-60.

10. Anderson ES, Datta N. Resistance to penicillins and its transfer in Enterobacteriaceae. Lancet 1965; 1:407-9.

11. Chabbert YA, Baudens JG. Transmissible resistance to six groups of antibiotics in Salmonella infections. Antimicrob Agents Chemother (Bethesda) 1965; 5:380-3. 
12. Anderson ES. Drug resistance in Salmonella Typhimurium and its implications. $\mathrm{Br}$ Med J 1968; 3:333-9.

13. Anderson ES, Threlfall EJ, Carr JM, McConnell MM, Smith HR. Clonal distribution of resistance plasmid-carrying Salmonella Typhimurium, mainly in the Middle East. $J$ Hyg (Lond) 1977; 79:425-48.

14. Grindley JN, Nakada D. The nucleotide sequence of the replication origin of plasmid NTP1. Nucleic Acids Res 1981; 9:4355-66.

15. Kelterborn E. Salmonella-species. First isolations, names and occurrence. SpringerVerlag, New York 1967.

16. European Food Safety Authority. The European Union summary report on trends and sources of zoonoses, zoonotic agents and food-borne outbreaks in 2015 http://onlinelibrary.wiley.com/doi/10.2903/j.efsa.2016.4634/epdf (accessed June 26, 2017). EFSA Journal 2016; 14:4634.

17. Le Hello S, Harrois D, Bouchrif B, et al. Highly drug-resistant Salmonella enterica serotype Kentucky ST198-X1: a microbiological study. Lancet Infect Dis. 2013; 13:672-9.

18. Ahmer BM, Tran M, Heffron F. The virulence plasmid of Salmonella typhimurium is self-transmissible. J Bacteriol 1999; 181:1364-8.

19. Fabre L, Zhang J, Guigon G, et al. CRISPR typing and subtyping for improved laboratory surveillance of Salmonella infections. PLoS One 2012; 7:e36995.

20. Mather AE, Reid SW, Maskell DJ, et al. Distinguishable epidemics of multidrugresistant Salmonella Typhimurium DT104 in different hosts. Science 2013; 341:15147. 
21. Petrovska L, Mather AE, AbuOun M, et al. Microevolution of Monophasic Salmonella Typhimurium during Epidemic, United Kingdom, 2005-2010. Emerg Infect Dis 2016; 22:617-24.

22. Kuijpers LM, Le Hello S, Fawal N, et al. Genomic analysis of Salmonella enterica serotype Paratyphi A during an outbreak in Cambodia, 2013-2015. Microb Genom 2016; 2:e00092.

23. Bankevich A, Nurk S, Antipov D, et al. SPAdes: a new genome assembly algorithm and its applications to single-cell sequencing. J Comput Biol 2012; 19:455-77.

24. Partridge SR, Hall RM. Evolution of transposons containing blaTEM genes. Antimicrob Agents Chemother 2005; 49:1267-8.

25. Kingsley RA, Msefula CL, Thomson NR, et al. Epidemic multiple drug resistant Salmonella Typhimurium causing invasive disease in sub-Saharan Africa have a distinct genotype. Genome Res 2009 Dec; 19:2279-87.

26. Marston CK, Hoffmaster AR, Wilson KE, et al. Effects of long-term storage on plasmid stability in Bacillus anthracis. Appl Environ Microbiol 2005; 71:7778-80.

27. Gounelle H, Szakvary A. [Antibiotics and food. IV. Use of antibiotics outside medical prescriptions]. Bull Acad Natl Med 1966; 150:149-56. In French.

28. Webb KE Jr, Fontenot JP. Medicinal drug residues in broiler litter and tissues from cattle fed litter. J Anim Sci 1975; 41:1212-7.

29. Threlfall EJ. Epidemic Salmonella Typhimurium DT 104-a truly international multiresistant clone. J Antimicrob Chemother 2000; 46:7-10.

30. Hopkins KL, Kirchner M, Mevius DJ. Multiresistant Salmonella enterica serovar 4,[5],12:i:- in Europe: a new pandemic strain? Euro Surveill 2010; 15:19580. 
Figure 1:

Figure 2:

Figure 3 : 